\title{
Screening of FDA approved drugs against COVID-19 Main Protease: Coronavirus disease
}

\author{
Vijayakumar Balakrishnan ${ }^{1 \star}$ and Karthik Lakshminarayanan² \\ 1 São Carlos Institute of Physics (IFSC), University of São Paulo, Av. Joao Dagnone, 1100 - Jardim \\ Santa Angelina, SãoCarlos 13563-120, Brazil. \\ ${ }^{2}$ Toxiven Biotech, Siva Nagar, Kovaipudhur, Tamil Nadu, India - 641042. \\ *Correspondence: vijaybioscience@ifsc.usp.br, vijaybioscience@gmail.com.
}

\begin{abstract}
In the end of December 2019, a new strain of coronavirus was identified in the Wuhan city of Hubei province in China. Within a shorter period of time, an unprecedented outbreak of this strain was witnessed over the entire Wuhan city. This novel coronavirus strain was later officially renamed as COVID-19 (Coronavirus disease 2019) by the World Health Organization. The mode of transmission had been found to be human-to-human contact and hence resulted in a rapid surge across the globe where more than 1,100,000 people have been infected with COVID-19. In the current scenario, finding potent drug candidates for the treatment of COVID-19 has emerged as the most challenging task for clinicians and researchers worldwide. Identification of new drugs and vaccine development may take from a few months to years based on the clinical trial processes. To overcome the several limitations involved in identifying and bringing out potent drug candidates for treating COVID-19, in the present study attempts were made to screen the FDA approved drugs using High Throughput Virtual Screening (HTVS). The COVID-19 main protease (COVID-19 Mpro) was chosen as the drug target for which the FDA approved drugs were initially screened with HTVS. The drug candidates that exhibited favorable docking score, energy and emodel calculations were further taken for performing Induced Fit Docking (IFD) using Schrodinger's GLIDE. From the flexible docking results, the following four FDA approved drugs Sincalide, Pentagastrin, Ritonavir and Phytonadione were identified. In particular, Sincalide and Pentagastrin can be considered potential key players for the treatment of COVID-19 disease.
\end{abstract}

\section{Keywords}

COVID-19; FDA approved drugs; High Throughput Virtual Screening; Sincalide; Pentagastrin 


\section{Introduction}

A new strain of Coronavirus(s) (CoVs) was identified in the concluding weeks of December 2019 and emerged in an alarming outbreak in the Wuhan city of Hubei province in China [1]. Initially named 2019-nCoV, the World Health Organization (WHO) officially declared the outbreak of 2019-nCoV in China on January 30, 2020 [2]. Following the consultations with the World Organization for Animal Health and the Food and Agriculture Organization of the United Nations, WHO officially renamed this strain as COVID-19 on February 11, 2020 [3]. Owing to the human-to-human transmission by the end of February 2020 , over 90,000 people were found to be infected with COVID-19 among which as many as 3000 cases have succumbed to this deadly infection. This rapid surge in people being infected with COVID-19 has forced the WHO to declare this a Global Public Health Emergency on $4^{\text {th }}$ March 2020.

Belongs to the Nidovirales order, CoVs genome is comprised of linear and positive-sense RNA molecule [4]. The Coronaviridae family is further classified into four genera: Alpha, Beta, Delta and Gamma CoVs. Members of these families generally consist of three to four envelope proteins with the membrane protein being the most abundant one. As of date, seven coronaviruses have been identified that infect humans. The strains of HCoV-OC43 and HCoV-HKU1 from Betacoronavirus and HCoV-229E from Alphacoronavirus were found to be the causative agents for common colds as well as severe lower respiratory tract infections in the youngest and oldest age groups [5]. The pandemic COVID-19 strain was initially considered to be related to the Beta coronaviruses strain of SARS-CoV (2002 outbreak) and MERS-CoV (2012 outbreak). Later on, it was further concluded that COVID-19 is closely related to SARSCoV and genetically clusters within Beta coronavirus subgenus Sarbecovirus [6-8].

To date (April 4, 2020), more than 1,131,893 people were infected with COVID-19 worldwide among which about59,902 deaths have been reported. Among the total cases, around 838,355 were found to be active with 233,636 people were officially reported to be recovered fully from the infection. In the height of this crisis, the rate and scale of COVID-19 spreading have been considered to be far worse when compared to that of the previous coronaviral epidemics. Moreover, due to the lack of investigations pertaining to the treatment of COVID-19, no specific therapies have been identified for treating this dreadful infection [2]. A preliminary study using the combination of protease inhibitor lopinavir/ritonavir has been reported for the treatment of COVID-19infected patients [9]. This combination has been generally used for treating patients affected by human immunodeficiency virus (HIV). Several existing antiviral agents such as nucleoside analogues, neuraminidase inhibitors, tenofovir disoproxil (TDF) and lamivudine (3TC) have also been tested against pathogenic CoVs [9]. The main protease (Mpro)/chymotrypsin-like protease (3CLpro) and RNA polymerase of COVID-19 have been considered attractive drug targets for designing potent antiviral compounds. A study by Xu et al in 2020 indicated that among the tested compounds Nelfinavir was identified as the best potential inhibitor against COVID-19 Mpro[10]. Similarly, a study by Yu-Chuan et al suggested that the nucleoside analogue Remdesivir was found effective against COVID-19 Mpro [11]. However, as these findings remain unapproved, results from these preliminary studies cannot be applied for therapeutic use and in the subsequent clinical setting for the treatment of COVID-19-infected patients [9,12].

In order to overcome the limitations prevailing in identifying potent antiviral drugs, the present study describes a combined molecular modeling approach carried out against COVID-19 Mpro. For this, the complete list of FDA approved drugs ( 1500 compounds) were retrieved from the Binding Database (BindingDB). Using the HTVS, drugs with favorable docking score, glide energy, glide emodel were further subjected to Induced Fit Docking (IFD) using Schrodinger's GLIDE. Finally, all the chosen drugs used in the IFD studies were analyzed for the favorable docking score, glide energy, glide emodel and interaction studies (hydrogen bond and hydrophobic) with COVID-19 Mpro. The following four drugsSincalide, Pentagastrin, Ritonavir and Phytonadione have exhibited better results with COVID-19 
Mpro. In particular, the potent inhibition of Sincalide and Pentagastrin drugs along with similar structurally related inhibitors could be used as a key player in the treatment of COVID-19 disease. The findings of the present study will provide other doctors/researchers with opportunities to treat the patients or identify the right drug to combat COVID-19.

\section{Methodology:}

Glide software (v11.1, Schrödinger, 2017, USA) was used for the molecular modeling calculations involving rigid (High Throughput Virtual screening) and flexible (Induced Fit Docking) docking studies. Chimera [13] and glide ligand interaction display software were used for graphical visualization, analysis of the hydrogen bond interaction and hydrophobic interactions between the active site amino acids and FDA drugs.

\section{Protein Preparation:}

The crystal structure of COVID-19 Mproin complex with N3inhibitor (6LU7) was retrieved from PDB (www.rcsb.org). The protein has three domains like I (residues 8-101), II (residues 102-184) and III (residues 201-303). The N3 inhibitor maintained the highest numbers of $\mathrm{H}$-Bond interactions with domain II (active site residues) [14]. The raw complex structure retrieved from PDBwas not suitable for the screening of FDA approved drugs using HTVs and IFD molecular modeling studies. In this connection, protein preparation (Schrödinger modules) was employed to fix the missed out information of bond orders, topologies, formal atomic charges, ionization, tautomeric states and removed unwanted water molecules in the structure. Moreover, Non-hydrogen atoms were energy minimized until the average root mean square deviation reaches $0.3 \AA$ and the amino acid flips were assigned.

\section{Drug selection:}

FDA-approved drugs( 1500 compounds)were recovered from the BindingDB with 3D conformations (https://www.bindingdb.org/bind/ByFDAdrugs.jsp). All these drugs were clustered within different categories such as peptide drugs, anti-HIV, anti-malarial, anti-Hepatitis C virus, anti-bacterial, anti-fungal, anti-inflammatory, natural source drugs and various other categories. In addition to this, all these drugs have also contained reliable molecular information with added hydrogens, 3D conversion, realistic bond lengths and bond angles, low energy structure with correct chirality's, ionization states, tautomers, stereochemistries and ring conformation.

\section{High Throughput Virtual screening (HTVs):}

HTVs is one of the methods for screening multiple compounds against active site key residues in the previously calculated grid receptor from a set of compounds. The energy minimized complex of COVID-19 Mproin complex with an inhibitor N3 was loaded in the workspace; the active site of the receptor was calculated automatically by picking the N3 in the receptor grid generation process. This process of docking the drug at the grid box (active site) yields a more accurate scoring function and energy of the protein-ligand complex. The docking score, glide energy, glide emodel and non-bonded interactions were analyzed in the HTVs results.

\section{Induced Fit Docking studies:}

In IFD (flexible docking), the proteins undergo side-chain or backbone conformation or both in many cases when a drug binds at the active site cavity. These conformational changes are one of the key processes to predict concomitant structural movements and accurate drug binding in the active site of the receptor. The best 4 drugs chosen from the HTVs results were further subjected to IFD studies. These drugs were redocked at the active site of the target. Docking score, glide energy, and glide emodel were obtained from the energetically favorable docked complexes and the best poses were chosen for analyzing the hydrogen bond and hydrophobic interactions. 


\section{Results and Discussion:}

\section{Anti- HIV drugs}

Anti-HIV drugs of Ritonavir, Indinavir and Lopinavir were redocked at the active site of the target using HTVs (Table-1). Based on the HTVS results, among the three drugs, Ritonavir alone taken for further IFD studies. The IFD docked complex of Ritonavir has exhibited a docking score -11.292 , glide energy -81.842 and glide emodel -128.743 (Figure 1; Table-2; Figure S1). The drug also maintains nonbonded interactions with the active site residues.

\section{Peptide drugs}

Sincalide/Cholecystokinin(CCK-8) is a C-terminal octapeptide that is naturally occurring in gastrointestinal peptide hormone. The Sincalide drug was bound well at the active site of COVID-19 Mpro. This IFD complex has possessed docking score -14.183 , glide energy -114.183 , and glide emodel 220.008 (Figure $1 \& 2$; Table-2) and has very good non-bonded interactions with the active site residues. It has been well established that the Potato protease inhibitors increases the circulation of cholecystokinin levels through a trypsin-dependent mechanism [15].

Pentagastrin is a synthetic polypeptide that was bound at the active site of COVID-19 Mpro. The IFD complex has docking score -15.316, glide energy -103.681, and glide emodel -195.461(Figure 1; Table-2; Figure S2)and has very good non-bonded interactions with the active site residues.

From the IFD results obtained for Sincalide and Pentagastrin, we hypothesis that an active site amino acid residue at cystine 145 can make disulfide bond interactions with a sulfur atom in the two peptide drugs. This hypothesis should be confirmed by biophysical and biochemical studies in the future.

\section{Natural source drugs (Vitamin K)}

Dicoumarol and Phytonadione (Vitamin K1)are vitamins. These drugs were redocked at the active site of the target in HTVs (Table-1). The IFD docked complex of Phytonadione has a docking score 9.578, glide energy -57.931 and glide emodel -84.813 (Figure 1; Table-2; Figure S3). The drug maintains non-bonded interactions with the active site residues. Phytonadione is rich in Green leafy vegetables. Medical expertise can suggest this nutritional diet for COVID -19 affected patients based on further reconfirmation.

\section{Other Drugs:}

The remaining drug candidates with proven properties such as anti-malarial, anti-Hepatitis C virus, anti-bacterial, anti-fungal, anti-inflammatory were also found to bound at the active site of COVID19 Mpro (Table-1). The drugs have maintained non-bonded interactions with the active site residues.

Table 1: Docking score, glide energy and glide emodel in SP docked complexes

\begin{tabular}{|l|l|l|l|l|l|l|l|}
\hline FDA drug & $\begin{array}{l}\text { Docking } \\
\text { score }\end{array}$ & $\begin{array}{l}\text { Glide } \\
\text { energy } \\
\text { (Kcal/mol) }\end{array}$ & $\begin{array}{l}\text { Glide } \\
\text { emodel } \\
\text { (Kcal/mol) }\end{array}$ & FDA drug & $\begin{array}{l}\text { Docking } \\
\text { score }\end{array}$ & $\begin{array}{l}\text { Glide } \\
\text { energy } \\
\text { (Kcal/mol) }\end{array}$ & $\begin{array}{l}\text { Glide } \\
\text { emodel } \\
\text { (Kcal/mol) }\end{array}$ \\
\hline N3 ${ }^{*}$ & -11.27 & -80.96 & -125.63 & CIPROFLOXACIN & -7.628 & -43.969 & -63.051 \\
\hline SINCALIDE & -12.09 & -93.783 & -152.565 & INDOMETHACIN & -7.653 & -49.226 & -71.042 \\
\hline PENTAGASTRIN & -11.225 & -83.516 & -141.213 & TELAPREVIR & -8.29 & -63.439 & -92.735 \\
\hline RITONAVIR & -11.644 & -81.385 & -131.095 & BOCEPREVIR & -6.181 & -50.987 & -65.677 \\
\hline INDINAVIR & -8.409 & -58.818 & -80.42 & FLUCONAZOLE & -7.859 & -46.243 & -66.068 \\
\hline LOPINAVIR & -8.148 & -63.668 & -89.424 & MICONAZOLE & -6.999 & -50.924 & -68.483 \\
\hline MEFLOQUINE & -7.454 & -41.733 & -56.069 & DICUMAROL & -6.202 & -40.023 & -52.402 \\
\hline AMODIAQUINE & -6.434 & -43.429 & -59.249 & PHYTONADIONE & -5.313 & -43.995 & -55.586 \\
\hline
\end{tabular}

${ }^{*}$ in the clinical trial 


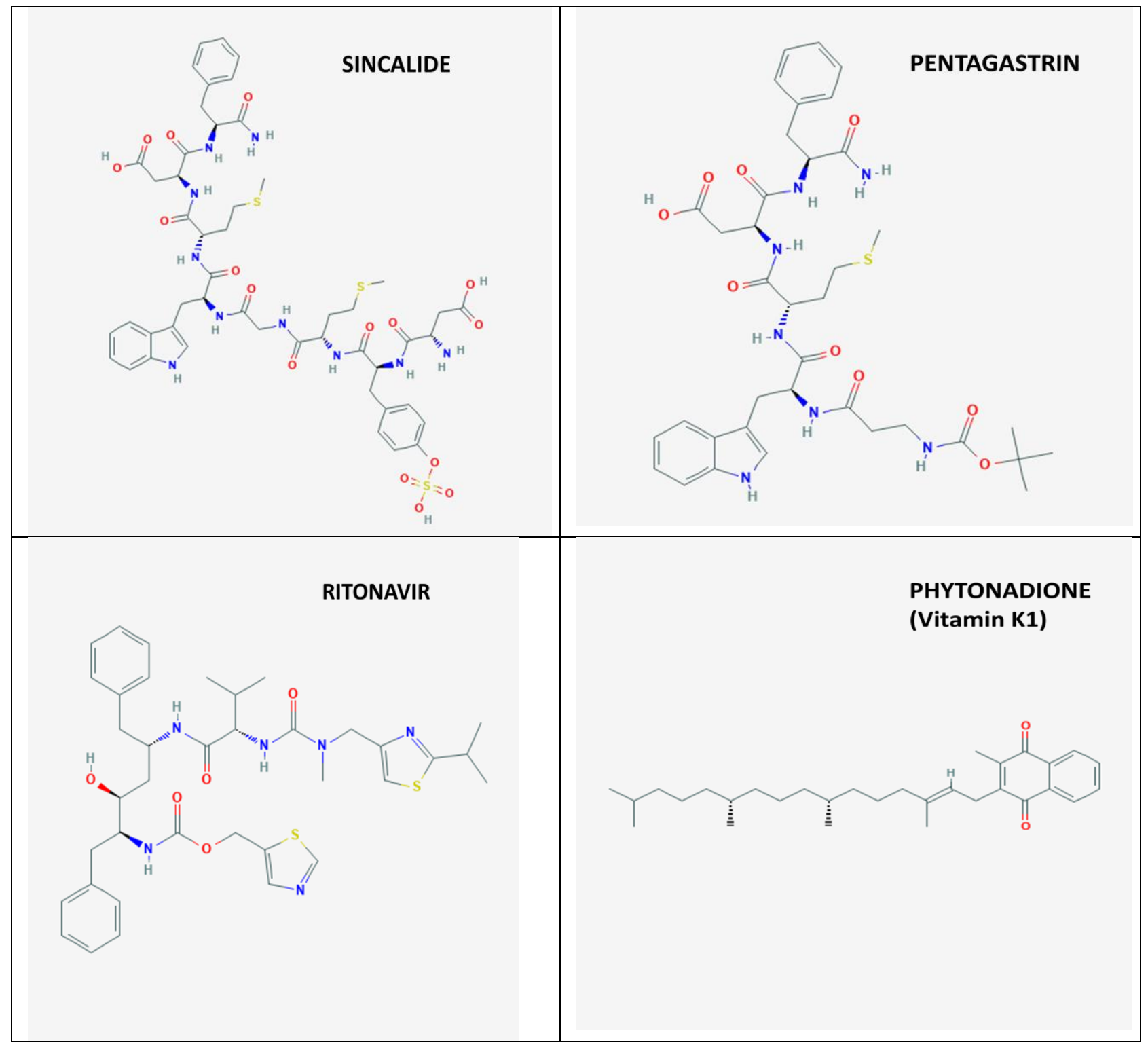

Figure 1: 2-D Chemical diagrams of Sincalide, Pentagastrin, Ritonavir and Phytonadione (Vitamine $\mathrm{K}_{1}$ ) 


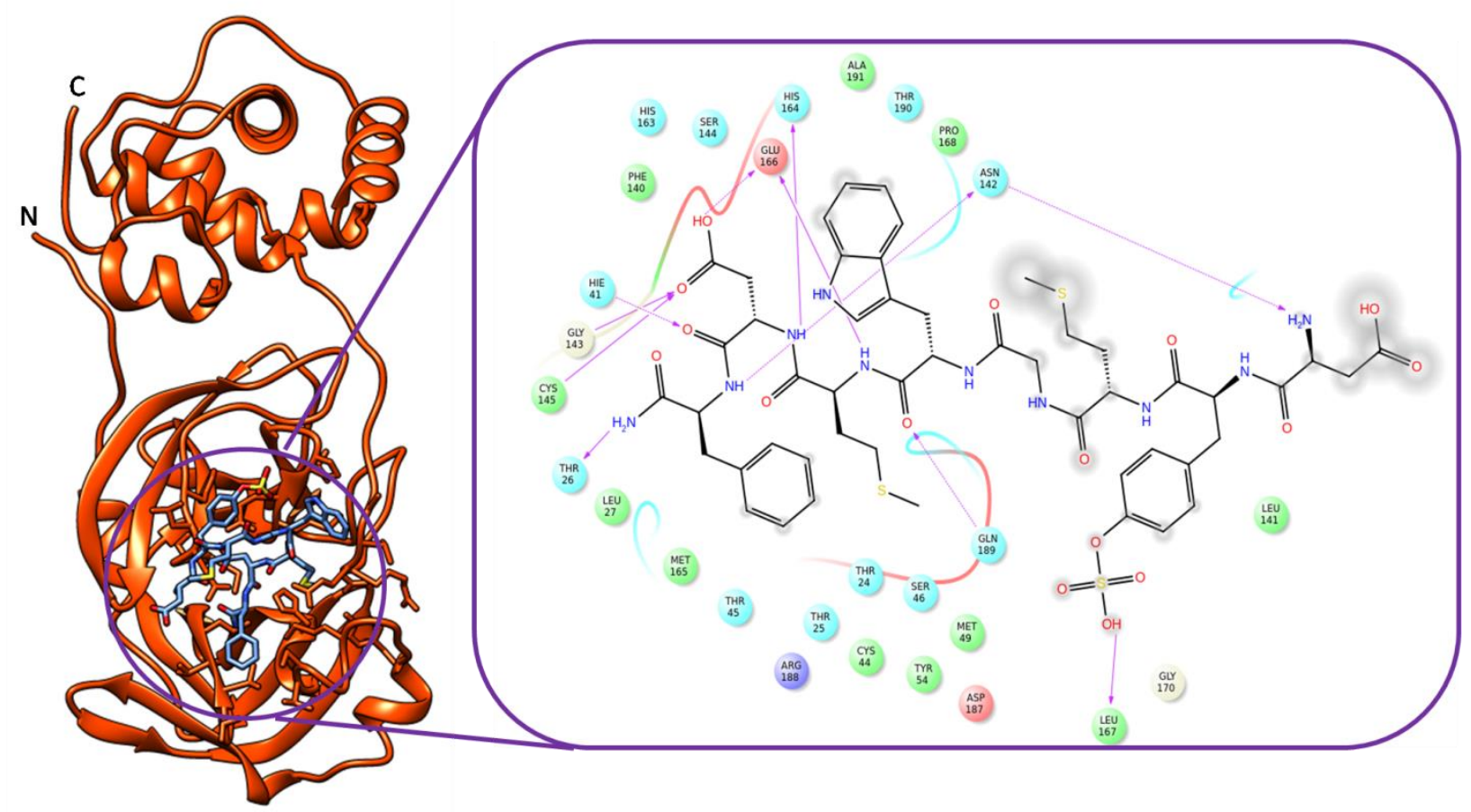

Figure 2: Hydrogen bond interactions of Sincalide with active site residues

Table 2: Docking score, glide energy and glide emodel in Induced fit docked complexes

\begin{tabular}{|l|l|l|l|}
\hline FDA drug & $\begin{array}{l}\text { Docking } \\
\text { score }\end{array}$ & $\begin{array}{l}\text { Glide } \\
\text { energy } \\
\text { (Kcal/mol) }\end{array}$ & $\begin{array}{l}\text { Glide } \\
\text { emodel } \\
\text { (Kcal/mol) }\end{array}$ \\
\hline N3 * & -13.145 & -88.302 & -152.882 \\
\hline SINCALIDE & -14.183 & -114.183 & -220.008 \\
\hline PENTAGASTRIN & -15.316 & -103.681 & -195.461 \\
\hline RITONAVIR & $-\mathbf{1 1 . 2 9 2}$ & -81.842 & -128.743 \\
\hline PHYTONADIONE & -9.578 & -57.931 & -84.813 \\
\hline
\end{tabular}

* in the clinical trial

\section{Conclusion:}

In this study, FDA-approved ( 1500 compounds) drugs were screened and based on the results, four drugs were compared with N3-redockedCOVID-19 Mproin the complex. The docking score, glide energy, glide emodel, hydrogen bond and hydrophobic interactions were analyzed. The four drugs Sincalide, Pentagastrin, Ritonavir and Phytonadionehave been found to possess better results with COVID-19 Mpro(Coronavirus disease). In particular, the potent inhibition exhibited by the FDA approved Sincalide andPentagastrindrugswere found to be more efficient in terms of docking score, energy and capability of forming hydrogen bond interactions with the active site residues. Altogether, the FDA approved drugs identified in the present study can be utilized in combinatorial therapy where these drugs, 
as well as inhibitors structurally similar to these peptide drugs, can be used in combination for the treatment of COVID-19 disease.

\section{ACKNOWLEDGMENT}

This work was supported by the National Institute of Science and Technology -Biorational Control of Insects and Pests (INCT-CBIP; grant numbers FAPESP 2008/51859-5 and CNPq 573742/2008-1), São Paulo State Foundation for Research (FAPESP; grant numbers 2018/00492-4. The authors would also like to thank Prof. Dr. Andre Luis Berteli Ambrosio and Prof. Dr. Rafael Victório Carvalho Guido for the research support and software facility.

\section{Conflict of interest}

Nil.

\section{References:}

1. Lee PI, Hsueh PR.Emerging threats from zoonotic coronaviruses-from SARS and MERS to 2019nCoV," J. Microbiol. Immunol. Infect.,2020; 1-3, doi: 10.1016/j.jmii.2020.02.001.

2. Rodríguez-Morales AJ, MacGregor K, Kanagarajah S, Patel D, Schlagenhauf P.Going global Travel and the 2019 novel coronavirus.Travel Med. Infect. Dis.,2020;33. doi: https://doi.org/10.1016/j.tmaid.2020.101578.

3. World Health Organization (WHO). Novel Coronavirus (2019-nCoV)," WHO Bull., no. JANUARY, 2020; 1-5, https://www.who.int/docs/default-source/coronaviruse/situation-reports/20200121sitrep-1-2019-ncov.pdf

4. Viruses ICoTo. ICTV 9th Report 2011 [14 January 2020]. Available from: https://talk.ictvonline.org/ictv-reports/ictv_9th_report/positive-sense-rna-viruses-2011/w/posrna_ viruses/223/coronaviridae-figures.

5. Yin Y, Wunderink RG. MERS, SARS and other coronaviruses as causes of pneumonia. Respirology. 2018;23(2):130-7.

6. Wuhan City Health Committee (WCHC). Wuhan Municipal Health Committee's report on unexplained viral pneumonia 2020 [14 January 2020]. Available from: http://wjw.wuhan.gov.cn/front/web/showDetail/2020010509020.

7. WHO. WHO Statement Regarding Cluster of Pneumonia Cases in Wuhan, China Geneva2020 [updated 9 January 202014 January 2020]. Available from: https://www.who.int/china/news/detail/09-01-2020-who-statement-regarding-cluster-ofpneumonia-cases-in-wuhan-china.

8. WHO. Novel Coronavirus - China Geneva2020 [updated 12 January 202014 January 2020]. Available from: https://www.who.int/csr/don/12-january-2020-novel-coronavirus-china/en/.

9. Lu H. Drug treatment options for the 2019-new coronavirus (2019-nCoV).Biosci Trends. 2020; 14(1):69-71. doi: 10.5582/bst.2020.01020. 
10. Zhijian Xu, Cheng Peng, Yulong Shi, Zhengdan Zhu, Kaijie Mu, Xiaoyu Wang, Weiliang Zhu. Nelfinavir was predicted to be a potential inhibitor of 2019-nCov main protease by an integrative approach combining homology modelling, molecular docking and binding free energy calculation. bioRxiv.org.,2020;https://doi.org/10.1101/2020.01.27.921627

11. Chang, Y.; Tung, Y.; Lee, K.; Chen, T.; Hsiao, Y.; Chang, H.; Hsieh, T.; Su, C.; Wang, S.; Yu, J.; Shih, S.; Lin, Y.; Lin, Y.; Tu, Y.E.; Hsu, C.; Juan, H.; Tung, C.; Chen, C. Potential Therapeutic Agents for COVID-19 Based on the Analysis of Protease and RNA Polymerase Docking. Preprints 2020; 2020020242 (doi: 10.20944/preprints202002.0242.v2).

12. Michelle L. Holshue, M.P.H., Chas DeBolt, M.P.H., Scott Lindquist, M.D., Kathy H. Lofy, M.D., John Wiesman, Dr. P.H., Hollianne Bruce, M.P.H., Christopher Spitters, M.D., Keith Ericson, P.A.-C., Sara Wilkerson, M.N., Ahmet Tural, M.D., George Diaz, M.D., Amanda Cohn, M.D., et al., First Case of 2019 Novel Coronavirus in the United States. N Engl J Med. 2020;382:92936.doi: 10.1056/nejmoa2001191.

13. Pettersen EF1, Goddard TD, Huang CC, Couch GS, Greenblatt DM, Meng EC, Ferrin TE.UCSF Chimera--a visualization system for exploratory research and analysis.J Comput Chem. 2004; 25(13):1605-12. https://doi.org/10.1002/jcc.20084

14. Zhenming Jin, Xiaoyu Du, Yechun Xu, Yongqiang Deng, Meiqin Liu, Yao Zhao, Bing Zhang, Xiaofeng Li, Leike Zhang, Chao Peng, YinkaiDuan, Jing Yu, Lin Wang, Kailin Yang, Fengjiang Liu, Rendi Jiang, Xinglou Yang, Tian You, Xiaoce Liu, Xiuna Yang, Fang Bai, Hong Liu, Xiang Liu, Luke W. Guddat, Wenqing Xu, Gengfu Xiao, Chengfeng Qin, Zhengli Shi, Hualiang Jiang, Zihe Rao, Haitao Yang. Structure of Mpro from 1 COVID-19 virus and discovery of its inhibitors. biorxiv.org.2020; doi: https://doi.org/10.1101/2020.02.26.964882

15. Komarnytsky S, Cook A and Raskin I. Potato protease inhibitors inhibit food intake and increase circulating cholecystokinin levels by a trypsin-dependent mechanism. Int J Obes (Lond). 2011; 35(2): 236-243.doi: 10.1038/ijo.2010.192 